\title{
An in vivo selection method to optimize trans-splicing ribozymes
}

\author{
KAREN E. OLSON and ULRICH F. MÜLLER ${ }^{1}$ \\ Department of Chemistry and Biochemistry, University of California, San Diego, La Jolla, California 92093, USA
}

\begin{abstract}
Group I intron ribozymes can repair mutated mRNAs by replacing the $3^{\prime}$-terminal portion of the mRNA with their own $3^{\prime}$-exon. This trans-splicing reaction has the potential to treat genetic disorders and to selectively kill cancer cells or virus-infected cells. However, these ribozymes have not yet been used in therapy, partially due to a low in vivo trans-splicing efficiency. Previous strategies to improve the trans-splicing efficiencies focused on designing and testing individual ribozyme constructs. Here we describe a method that selects the most efficient ribozymes from millions of ribozyme variants. This method uses an in vivo rescue assay where the mRNA of an inactivated antibiotic resistance gene is repaired by trans-splicing group I intron ribozymes. Bacterial cells that express efficient trans-splicing ribozymes are able to grow on medium containing the antibiotic chloramphenicol. We randomized a 5 '-terminal sequence of the Tetrahymena thermophila group I intron and screened a library with $9 \times 10^{6}$ ribozyme variants for the best trans-splicing activity. The resulting ribozymes showed increased trans-splicing efficiency and help the design of efficient trans-splicing ribozymes for different sequence contexts. This in vivo selection method can now be used to optimize any sequence in trans-splicing ribozymes.
\end{abstract}

Keywords: in vivo selection; group I intron; ribozyme; trans-splicing

\section{INTRODUCTION}

Group I intron ribozymes are catalytic RNAs (ribozymes) that can splice themselves out of primary transcripts, many of them without the help of proteins (Kruger et al. 1982). In contrast to these naturally cis-splicing ribozymes, manmade variants can catalyze trans-splicing reactions (for a review, see Fiskaa and Birgisdottir 2010). The trans-splicing group I introns in our study lack a $5^{\prime}$-exon, and their recognition sequence is modified such that the ribozyme can base pair to a target site on the substrate RNA. In this format, the ribozyme replaces the 3 '-fragment of the substrate RNA downstream from the splice site, with its own 3 '-exon (Fig. 1). This replacement can repair an mRNA if the mRNA carries a mutation in its $3^{\prime}$-fragment and the ribozyme $3^{\prime}$-exon is the sequence of the mRNA $3^{\prime}$ fragment without mutation (Sullenger and Cech 1994). By being able to change the sequence of specific mRNAs, trans-splicing ribozymes take an important place in the toolbox of RNAs that can be used to alter the RNA

\footnotetext{
${ }^{1}$ Corresponding author.

E-mail ufmuller@ucsd.edu.

Article published online ahead of print. Article and publication date are at http://www.rnajournal.org/cgi/doi/10.1261/rna.028472.111.
}

inventory of a cell: The degradation of specific cellular RNAs can be affected by siRNAs, hammerhead ribozymes, HDV ribozymes, and the RNase $\mathrm{P}$ ribozyme. In contrast, trans-splicing ribozymes can alter the sequence of specific cellular RNAs. The alteration of RNA sequences in the cell could be used to repair mRNAs in the treatment of genetic disorders (Sullenger and Cech 1994) and for the specific killing of cells that express viral RNAs (Ayre et al. 1999; Ryu et al. 2003) and of cancer cells by targeting their increased telomerase reverse transcriptase mRNA (Kwon et al. 2005). Cell death is accomplished by a toxic peptide that is encoded in the transferred 3'-exon (Ayre et al. 1999).

Current trans-splicing group I intron ribozymes are not efficient enough in vivo for therapeutic applications. One way to increase the level of trans-splicing products in vivo is to raise the ribozyme expression levels, which can facilitate the repair of $10 \%-50 \%$ of a specific mRNA in cells under ideal conditions (Jones and Sullenger 1997; Lan et al. 1998; Byun et al. 2003). However, the high ribozyme expression levels necessary for these efficiencies are not applicable in a therapeutic setting. Our aim is to improve the in vivo repair efficiency with the aim that low ribozyme concentrations can achieve sufficient levels of mRNA repair.

Previous approaches to improve the trans-splicing efficiency were mostly based on designing and testing individual 


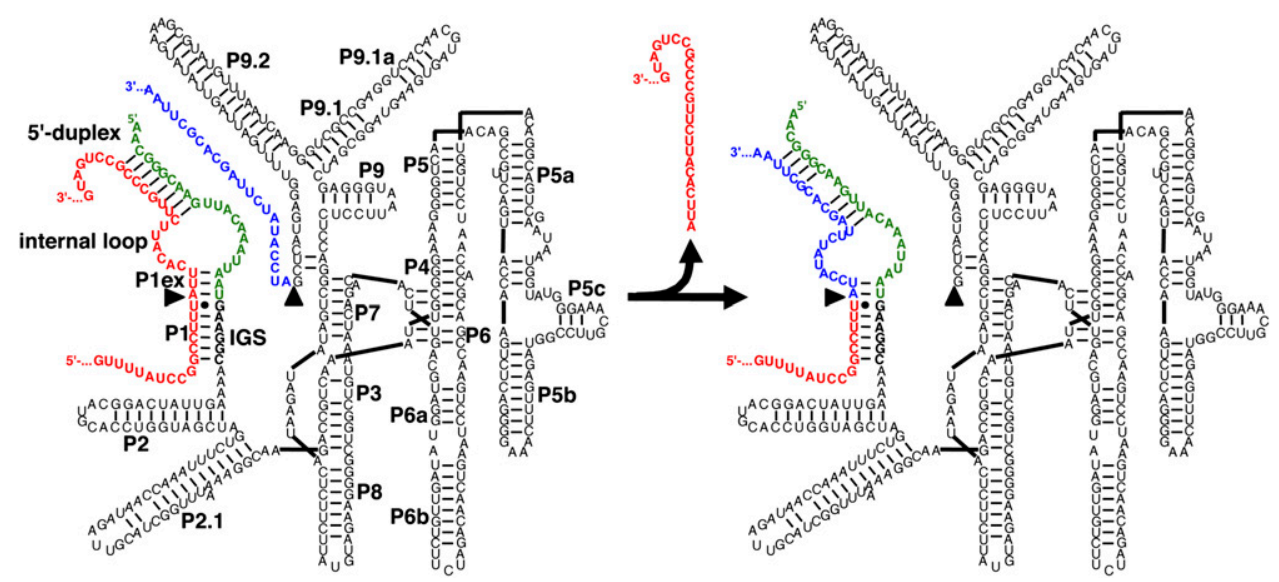

FIGURE 1. Secondary structure of the trans-splicing ribozyme construct used in this study, and its change during trans-splicing. The ribozyme is shown in gray, with the duplexes labeled. Note that the P4-P6 domain is shown to the right of the catalytic core for clarity. The mRNA is shown in red, with the splice site marked by an arrowhead. The EGS is shown in green. The ribozyme $3^{\prime}$-exon is shown in blue, with the $3^{\prime}$-splice site marked with an arrowhead. The sequence of the EGS used in this figure is that of clone R3C7, which was a result of the in vivo selection. Secondary structures formed by the EGS are labeled as 5'-duplex, internal loop, P1ex (P1 extension), and P1 helix. The reaction from substrate (left) to product (right) is indicated by arrows.

ribozyme constructs (Kohler et al. 1999; Ayre et al. 2002; Byun et al. 2003). In contrast, combinatorial approaches can test millions of ribozymes in parallel. Combinatorial approaches were crucial for the development and optimization of many ribozymes that would not have been found by design (for review, see Ellington et al. 2009). However, group I intron ribozymes present a challenge for combinatorial systems because the ribozymes separate themselves from their products, which prevents the traditional selection of the catalyst via the product. One elegant solution to this problem is to select these ribozymes based on their potential to catalyze the reverse reaction, thereby linking themselves with reaction products (Beaudry and Joyce 1992; Treiber et al. 1998; Ohuchi et al. 2004). However, the resulting ribozymes were inefficient in catalyzing the complete splicing process because they were selected to catalyze only one of the two catalytic steps (Ohuchi et al. 2004).

To optimize trans-splicing ribozymes for the complete splicing reaction in a combinatorial approach, we developed an in vivo selection method, which compartmentalizes the ribozyme with its substrate and product in cells. Combinatorial methods have also been used in vivo but did not employ the powerful combination with a ribozyme library of high complexity and multiple rounds of selection. Previous studies used fluorescence activated cell sorting (FACS) to select trans-splicing ribozymes that repair the mRNA of a fluorescent protein in mammalian cells (Hasegawa et al. 2004), used histidine prototrophy to select trans-splicing ribozymes that activate a metabolic pathway in yeast cells (Ayre et al. 2002), or used the resistance to the antibiotic kanamycin to detect excision of a cis-splicing ribozyme from the kanamycin nucleotidyl transferase mRNA in Escherichia coli (Guo and Cech 2002). In addition to the compartmentalization of ribozyme and substrate, the in vivo format is advantageous because several ribozymes, including the Tetrahymena group I intron ribozyme, show differences between in vitro and in vivo activity (Coetzee et al. 1994; Nikolcheva and Woodson 1999; Mahen et al. 2005), and the ultimate goal for trans-splicing ribozymes is a high activity in vivo.

Here we report an in vivo selection method that selects the best trans-splicing ribozymes from millions of ribozyme variants. The method employs ribozyme libraries with high complexity, uses several rounds of enrichment, and is based on materials that exist in most molecular biology laboratories. A selection from millions of ribozyme variants can be completed by one person in $<3$ mo. We validated this method by optimizing the ribozyme $5^{\prime}$ terminus for a given splice site in the mRNA of chloramphenicol acetyl transferase (CAT). The method yielded $5^{\prime}$-terminal sequences that mediated increased trans-splicing efficiency. The robust in vivo selection technique now makes it possible to optimize any sequence in trans-splicing ribozymes.

\section{RESULTS}

\section{Identification of efficient splice sites}

Trans-splicing ribozymes can, in principle, target every uridine residue of a substrate RNA, but the target sites need to be accessible. To determine accessible splice sites on our model mRNA substrate, the mRNA of CAT, we first determined accessible splice sites by an established protocol, the trans-tagging assay (Lan et al. 1998). In this assay, the mRNA is incubated with trans-splicing ribozymes that carry a randomized internal guide sequence (IGS). This enables the ribozyme population to splice on every accessible site on the mRNA. The sites at which trans-splicing occurred were 
identified by RT-PCR, cloning, and sequencing. Thirteen different splice sites were identified on cat mRNA (Supplemental Table S1). We chose splice site 177 for all further experiments. The corresponding IGS for splice site 177 is $5^{\prime}$-GAAGGC-3'.

\section{Design of the ribozyme gene library}

In addition to targeting an accessible splice site, efficiently trans-splicing ribozymes require a $5^{\prime}$-terminal extension that forms specific secondary structures with the target mRNA (Fig. 1; Kohler et al. 1999). This $5^{\prime}$-terminal extension was termed the EGS (extended guide sequence). The secondary structures formed with the substrate mRNA are the P1 duplex, the P1 extension, an internal loop, a $5{ }^{\prime}$-duplex, and a P10 duplex. However, several details on this structure were still unclear. First, it was unclear how long the singlestranded portions on each side of the internal loop should be. Second, the optimal sequence in the single-stranded portion of the internal loop was unknown. Third, it was unclear whether the first base pair of the P1 extension duplex should be a C:A base pair instead of a U:A base pair, as suggested by one of our earlier selections (Supplemental Fig. S1).

To address these questions, we designed a library of ribozyme genes with six subpools (Fig. 2). Each of these subpools formed the $5^{\prime}$-duplex in a different register so that the selection could optimize the relative length of singlestranded nucleotides on each side of the internal loop. The $5^{\prime}$-duplex fixed the identity of 8 nucleotides (nt). The next $9 \mathrm{nt}$ in the EGS were randomized. This allowed optimizing the length of the $5^{\prime}$-duplex because selected nucleotides could extend the $5^{\prime}$-duplex; it allowed optimizing the length of the P1 extension because selected nucleotides could extend the P1 extension duplex; and it allowed optimizing the sequence in the single-stranded portion of the EGS. The next 2 nt were fixed because they were known to be involved in the minimum 3-bp P1 extension duplex (Kohler et al. 1999; Guo and Cech 2002). Finally, the last nucleotide of the EGS and the first nucleotide of the P1 extension was

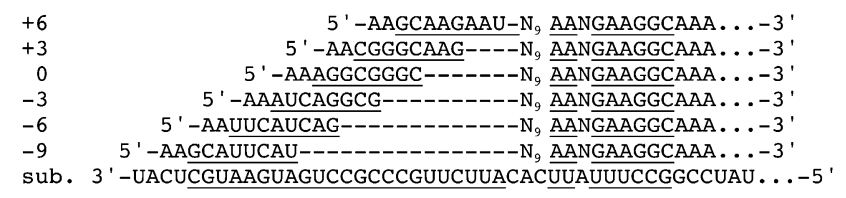

FIGURE 2. Design of the pool of EGSs for the in vivo selection. Six subpools with six different registers $(+6,+3,0,-3,-6,-9)$ were generated to represent six different geometries of the internal loop with the mRNA (bottom). The positions of randomized nucleotides are denoted with $\mathrm{N}$. Underlined characters show the position of duplexes between EGS and mRNA. All six subpools form identical P1 interactions at the IGS (GAAGGC) and at the P1 extension, which is constituted by the $3 \mathrm{bp}$ adjacent to the mRNA splice site. The six subpools differ in the position of their 8-bp 5'-duplex on the mRNA, formed with nucleotides 3-10 of the EGS. randomized to determine whether it should form a U:A or a C:A base pair with the mRNA.

\section{Generation of the plasmid library and the $E$. coli library}

The library of ribozyme genes with partially randomized EGSs was generated by PCR and cloned into a library plasmid (Supplemental Fig. S2). The ribozyme expression was driven by an attenuated version of the IPTG inducible trc promoter (Weiss et al. 1999). The promoter for bacteriophage T7 RNA polymerase, which was used in earlier studies (Sullenger and Cech 1994), was not chosen because it inhibited cell growth. The down-regulated trc promoter was chosen from three different variants of the trc promoter because it facilitated the best cell growth but expressed sufficient levels of ribozyme to show trans-splicing activity (data not shown).

The gene of the substrate mRNA (cat) was under the control of a constitutive promoter from its parent plasmid pLysS (Novagen). To allow the selection of mRNA repairing trans-splicing ribozymes, we introduced a frameshift deletion ( $\Delta \mathrm{G} 322)$ into the cat mRNA. This mutation abolished the chloramphenicol resistance such that $E$. coli cells carrying a plasmid with this mutated gene did not form detectable colonies at a chloramphenicol concentration of $2 \mu \mathrm{g} / \mathrm{mL}$ on culture plates. Transcription termination sequences were inserted after the cat gene and after the ribozyme gene to prevent read-through.

Each plasmid library was transformed into E. coli cells and plated on medium selecting for the presence of the library plasmid through ampicillin resistance. This resulted in at least $1.5 \times 10^{6}$ cells for each subpool. At this point, the six subpools were mixed, resulting in the E. coli library that was used for the selection. To test the composition of this initial pool (round 0 ), we sequenced 50 of its clones. The sequences confirmed that each of the six subpools was represented equally, within statistical variation (Supplemental Table S2).

\section{Progress of the in vivo selection}

To select those cells from the E. coli library that contained efficiently trans-splicing ribozymes, we induced the expression of ribozymes by IPTG and plated the library on SOC agar plates that contained IPTG and contained chloramphenicol at a concentration of $8 \mu \mathrm{g} / \mathrm{mL}$. This chloramphenicol concentration allowed the growth of $\sim 0.1 \%-1 \%$ of the plated cells. Cells that formed colonies were assumed to contain ribozymes that efficiently repaired the inactivated cat mRNA to mediate resistance against chloramphenicol. The number of cells plated on the selection plates was 9 million, which contained 1.5 million cells from each subpool. Because this covered the sequence space $\left(4^{10}\right)$ 1.43 -fold, we expected that $76 \%$ of all possible sequences were represented in this first round of the selection (for the calculation, see Materials and Methods). 
Five rounds of the selection were performed to eliminate false positives due to genomic mutations in E. coli or due to mutations in the library plasmid. After each selection step, the library plasmids were extracted from the selected cells. The ribozyme genes were isolated by restriction digest, agarose gel purification, and PCR and were ligated back into fresh library plasmid (Fig. 3). In each selective step after the first round, at least threefold more cells encoding a ribozyme were plated than the maximum complexity of the pool at that stage. Therefore, at least $95 \%$ of the pool complexity was maintained in these later selection rounds.

Three rounds of selection were sufficient to select the best EGSs. This was concluded based on the sequences of 20 clones that were collected in each round. These sequences showed that the enrichment of subpools had saturated after three rounds of selection (Fig. 4) and that most clones were found as multiplicates after three rounds (Table 1). Therefore, we considered clones from round 3, 4, and 5 as the winners of the selection. Most of the winners contained EGSs from the subpools $+6(30 / 60)$ and $+3(22 / 60)$. In addition, a few isolates stemmed from subpools $-3(5 / 60)$ and $-6(3 / 60)$, while no isolates from subpools -9 and 0 were found. The clones were named by the selection round $\mathrm{R}$ in which they were first detected and by a successive clone number $\mathrm{C}$, for example, R3C7.

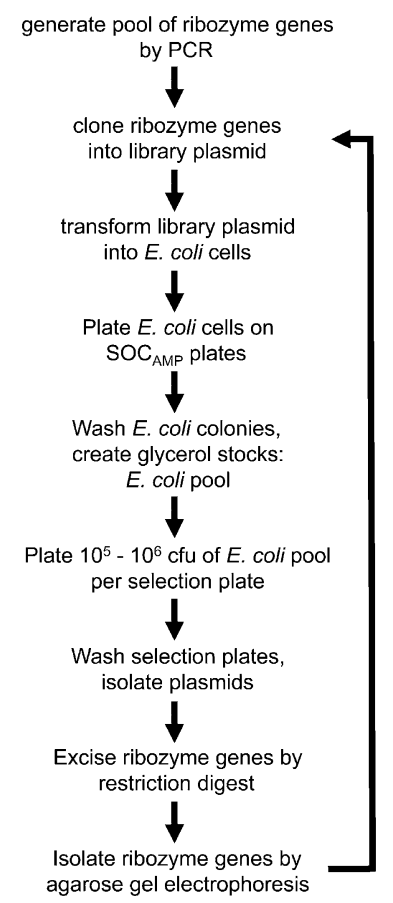

FIGURE 3. Schematic for the work flow of the in vivo selection procedure. $\mathrm{SOC}_{\mathrm{AMP}}$ plates refer to agar plates for bacterial growth, supplemented with the antibiotic ampicillin. Selection plates refer to agar plates for bacterial growth, supplemented with IPTG and the antibiotic chloramphenicol. More detailed descriptions are given in the text and in the Materials and Methods.
The early disappearance of subpools -9 and 0 may be due to the tendency of their respective EGSs to form selfstructures in the absence of a substrate mRNA. Using mfold, we predicted the average energy for self-structures, of all 50 sequences obtained from the starting population (Supplemental Table S2). Pools -9 and 0 showed the strongest self-structure energies $(-3.5 \pm 0.6 \mathrm{kcal} / \mathrm{mol}$ and $-5.1 \pm 0.8 \mathrm{kcal} / \mathrm{mol}$, respectively). The other subpools had self-structure energies of $-2.6 \pm 1.0 \mathrm{kcal} / \mathrm{mol}(+6),-3.1 \pm$ $0.8 \mathrm{kcal} / \mathrm{mol}(+3),-2.7 \pm 0.8 \mathrm{kcal} / \mathrm{mol}(-3)$, and $-1.3 \pm$ $0.4 \mathrm{kcal} / \mathrm{mol}(-6)$. Such self-structure formation would prevent the IGS and the EGS from pairing with the substrate, thereby reducing the trans-splicing efficiency and causing the disappearance of subpools -9 and 0 from the population.

\section{Trans-splicing efficiencies of selected EGSs in vivo}

To test whether the selection succeeded in isolating ribozymes that mediate high in vivo activity, we tested their trans-splicing efficiency in vivo. The eight most frequently selected sequences were chosen, as well as nine control sequences: four clones from the library before selection occurred, four clones that were designed based on published principles, and one clone without EGS (Table 1). Their in vivo trans-splicing efficiency was quantified by measuring the doubling times of E. coli cells expressing the chosen ribozymes and the inactivated cat gene in liquid medium containing chloramphenicol. All clones with selected ribozymes showed doubling times $<100 \mathrm{~min}$, whereas none of the clones with designed ribozymes showed doubling times $<100 \mathrm{~min}$ (Table 1). Sequencing confirmed that these clones did not differ in their internal ribozyme sequence from the wild-type sequence. These results showed that the selection was successful in isolating ribozymes with increased in vivo activity. The highest in vivo activities stemmed from ribozymes of the subpools $+3,-3$, and -6 . Interestingly, one preselected EGS (PS39) mediated good in vivo activity. Most preselected and designed EGSs showed lower in vivo activity than the ribozyme without EGS sequence (no EGS). This confirmed that nonoptimized EGSs inhibited mRNA repair in vivo, as it was found previously (Kohler et al. 1999).

We were surprised to find that the most frequently selected ribozyme (R3C14) did not mediate the fastest growth in liquid medium (Table 1). We hypothesized that this may be because the in vivo selection used growth on plates, whereas the doubling times were determined in liquid culture, which differ in their requirements on the cells. The difference between growth on plates and in liquid medium is caused by the diffusion of nutrients and oxygen into the bacterial colony (Reyrolle and Letelier 1979; Lewis and Wimpenny 1981), which limits the growth on plates but not in liquid culture. To test this hypothesis, we measured the growth of all clones for growth on plates containing chloramphenicol that were selected at least three times in the selection. The growth was judged by the average diameter of 


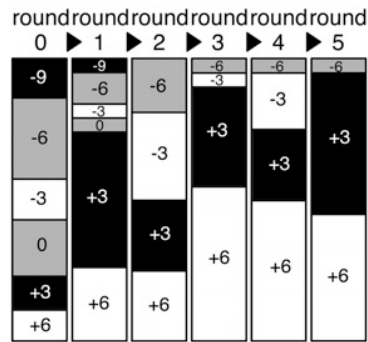

FIGURE 4. Enrichment of subpools in the ribozyme library, during five rounds of in vivo selection. Fifty clones were sequenced from the initial pool (round 0 ), and 20 clones were sequenced from each selected pool (pools 1-5). The relative abundance of each subpool is shown as the size as the corresponding rectangle. Note that subpools -9 and 0 disappeared within two rounds of selection. The corresponding numerical values are given in Supplemental Table S2.

their colonies (Fig. 5). The results showed that clone R3C14 grew better than any other clone on plates, which paralleled its frequency among the selected clones. This confirmed our hypothesis that the reason for the high enrichment of clone $\mathrm{R} 3 \mathrm{C} 14$ during the selection was its superior growth on plates. While these clones are not truly artifacts (they do facilitate faster growth than any of the designed clones), one could define the winners of the selection as the four sequences facilitating the fastest growth in liquid medium, representing 17 out of 60 clones.

The growth behaviors on plate and in liquid culture grouped the eight selected ribozymes into two classes (Fig. 5). One class contained clone $\mathrm{R} 3 \mathrm{C} 14$, which grew, on average, better on plate but not as well in liquid culture. The other class contained R3C7, which grew better in liquid culture but not as well on plates. Because the only difference between these ribozymes lies in their EGSs, we hypothesized that the secondary structures formed between the EGS and the substrate (Fig. 6A) or between the EGS and the product (Fig. 6B) caused the different growth behavior of the two classes. In support of this hypothesis, the predicted secondary structures showed a systematic difference: The class that contained clone $\mathrm{R} 3 \mathrm{C} 7$ formed an internal loop between the substrate and EGS with five or six singlestranded nucleotides on the mRNA side, but it did not form a conserved structure between product and EGS. In contrast, the class that contained clone R3C14 formed an internal loop between the trans-splicing product and EGS with five or six single-stranded nucleotides on the mRNA side but not between the substrate and EGS. If the length of five to six single-stranded nucleotides on the mRNA side of the internal loop represents an optimal geometry, then the two classes of ribozymes would use two different strategies to increase trans-splicing efficiency: The class containing R3C7 may optimize the interactions for the first step of splicing, whereas the class containing R3C14 may optimize the interactions for the second step of splicing. This hypothesis will be tested in future studies, with additional selections using different splice sites on the cat mRNA.
Two additional observations were made for the predicted secondary structures between EGS and substrate or product. First, the length of the P1 extension was 3 bp for all selected clones. The four clones that had $<3$ bp (designed clones Des 2 and Des4, and two clones from the preselection library, PS45 and PS66) showed poor in vivo repair efficiencies (Table 1). None of the selected clones formed a $\mathrm{P} 1$ extension $>3 \mathrm{bp}$, although the randomized sequence would have allowed the formation of longer P1 extensions. This suggests that the optimal length of the P1 extension is exactly $3 \mathrm{bp}$, consistent with previously reported results (Kohler et al. 1999). Second, none of the selected EGSs formed a P10 helix, that is, a duplex between the singlestranded region of the internal loop of the EGS with the $3^{\prime}$ exon. The two designed clones that formed a P10 helix (Des2 and Des4) showed low trans-splicing efficiency. Because the formation of a P10 helix increased the transsplicing efficiency in a different sequence context (Kohler et al. 1999; Byun et al. 2003), we assume that the beneficial effect of a P10 helix is dependent on the sequence context.

To test whether the growth rate of cells was a good measure for the trans-splicing efficiency in vivo, we measured the fraction of repaired mRNA in cells by quantitative RT-PCR (Fig. 7). Four ribozyme variants were chosen to cover the range of doubling times mediated by the ribozyme variants. This included the two EGSs that promoted the fastest doubling time in liquid medium ( $\mathrm{R} 3 \mathrm{C} 7$, register +3 ; $\mathrm{R} 3 \mathrm{C} 9$, register -6$)$, one that facilitated medium growth $(\mathrm{R} 3 \mathrm{C} 19$, register +6$)$, and one preselected EGS that facilitated only slow growth (PS72, register +6$)$. Growth in liquid culture was used for this correlation because the RNA samples for quantitative RT-PCR were prepared by growth in liquid medium. The quantitation of cat mRNA and trans-splicing products reported that between $0.4 \%$ and $3.8 \%$ of the mRNA was repaired by the trans-splicing ribozymes. The fraction of repaired mRNA and the doubling time of the cells were correlated well $(r=0.95)$. This suggests that the cell doubling time is a good measure for in vivo trans-splicing efficiency.

The selection system can now be used to improve the efficiency of any trans-splicing ribozyme. For example, trans-excision ribozymes (Dotson et al. 2008) or transinsertion ribozymes (Johnson et al. 2005) could be optimized with this system. Additionally, in combination with mutagenic PCR, the in vivo selection system can be converted to an in vivo evolution system: After each round of selection, new mutations can be introduced by mutagenic PCR (Cadwell and Joyce 1994) such that over multiple rounds, the population of evolving ribozymes successively accumulates beneficial mutations. This procedure would allow optimizing any sequence on the ribozyme, and the successive exploration of sequence space would facilitate the screening of far more functional sequences than can be tested in a selection experiment. We expect that such evolutions will facilitate even larger 
TABLE 1. Extended guide sequences (EGSs) that were used in this study

\begin{tabular}{lcccc}
\hline Clone & Loop sym. & $\begin{array}{c}\text { Clones } \\
\text { found }\end{array}$ & $\begin{array}{c}\text { Doubling } \\
\text { time }(\mathrm{min})\end{array}$ & Sequence \\
\hline NoEGS & - & - & $115 \pm 27$ & - \\
R3C14 & +6 & $19 x$ & $86 \pm 13$ & AAGCAAGAAUGUAGUACGUAAU \\
R3C7 & $+\mathbf{3}$ & $\mathbf{7 x}$ & $\mathbf{6 3} \pm \mathbf{3}$ & AACGGGCAAGUUACAAAUUAAU \\
R5C6 & +3 & $7 \mathrm{x}$ & $97 \pm 16$ & AACGGGCAAGUUAGAAUUUAAU \\
R2C16 & +3 & $4 \mathrm{x}$ & $93 \pm 4$ & AACGGGCAAGAAAAAAUUAAAU \\
R3C5 & $\mathbf{- 3}$ & $\mathbf{4 x}$ & $\mathbf{6 5} \pm \mathbf{5}$ & AAAUCAGGCGAGAUUACAAAAU \\
R3C3 & +6 & $3 \mathrm{x}$ & $88 \pm 10$ & AAGCAAGAAUGUAUACCACAAU \\
R3C4 & $+\mathbf{3}$ & $\mathbf{3 x}$ & $\mathbf{6 5} \pm \mathbf{5}$ & AACGGGCAAGUAAAGAAAUAAU \\
R3C9 & $\mathbf{- 6}$ & $\mathbf{3 x}$ & $\mathbf{6 1} \pm \mathbf{1}$ & AAUUCAUCAGAGGCAAGCAAAU \\
R3C15 & +6 & $2 \mathrm{x}$ & n.d. & AAGCAAGAAUGUAGCAAGUAAU \\
R3C11 & +6 & $2 \mathrm{x}$ & n.d. & AAGCAAGAAUGUUAUCAUCAAU \\
R3C19 & +6 & $1 \mathrm{x}$ & $90 \pm 13$ & AAGCAAGAAUUCAACAAAAAAU \\
R4C28 & +6 & $1 \mathrm{x}$ & n.d. & AAGCAAGAAUAGAACCUAAGAU \\
R5C19 & +6 & $1 \mathrm{x}$ & n.d. & AAGCAAGAAUAUUAUCGUCAAU \\
R3C6 & +6 & $1 \mathrm{x}$ & n.d. & AAGCAAGAAUGAUAUCUGGAAC \\
R3C12 & +3 & $1 \mathrm{x}$ & n.d. & AACGGGCAAGUGUUAGAUUAAU \\
R4C1 & -3 & $1 \mathrm{x}$ & n.d. & AAAUCAGGAGAGAAAAAUCAAU \\
Des1 & +6 & - & $171 \pm 34$ & AAGCAAGAAUAGAACCUAAGAU \\
Des2 & +6 & - & $113 \pm 24$ & AAGCAAGAAUAGACUGACGAAU \\
Des3 & -1 & - & $123 \pm 26$ & AACAGGCGGGAGAAUAUAAGAU \\
Des4 & -1 & - & $131 \pm 9$ & AACAGGCGGGAGACGUACGAAU \\
PS72 & +6 & - & $167 \pm 12$ & AAGCAAGAAUUCGGUUGCUAAC \\
PS45 & +3 & - & $119 \pm 9$ & AACGGGCAAGAAGGUAUCGAAU \\
PS39 & 0 & - & $68 \pm 4$ & AAAGGCGGGCUGCGUUUAAAAU \\
PS66 & -9 & - & $128 \pm 6$ & AAGCAUUCAUGAUAUCGAGAAU \\
\hline The & & - & &
\end{tabular}

The first column shows the clone name. Names starting with an $\mathrm{R}$ label clones that were identified during the in vivo selection, which indicates the selection round $R$ in which it appeared first and its clone number C. Ribozymes from the R3C7 class are in bold, and ribozymes from the class R3C14 are in italics. The prefix "Des" indicates that the EGS was designed, based on published principles. The prefix "PS" indicates that the EGS was from the preselection pool (round 0 ). The second column shows the symmetry of the internal loop, i.e., the register between mRNA strand and EGS in the $5^{\prime}$-duplex relative to the register at the IGS. The third column shows the number of clones that were found during the in vivo selection, as the sum of rounds 3,4 , and 5 . The fourth column shows the doubling time of cells expressing the ribozyme variant, in liquid medium, in the presence of chloramphenicol. n.d. indicates "not determined." Errors are SEMs from at least three independent experiments. The fifth column shows the sequence of the EGSs. Bold characters show nucleotides that were predicted to participate in the $5^{\prime}$-duplex (left) or the P1 extension duplex (right).

increases in trans-splicing efficiency and ultimately allow the application of trans-splicing ribozymes for therapeutic applications.

\section{DISCUSSION}

We developed an in vivo selection procedure to optimize the efficiency of trans-splicing ribozymes in vivo. The method was validated by selecting ribozymes with improved EGSs from a library with 9 million ribozyme variants. The in vivo selection method can now be used to optimize any sequence on trans-splicing ribozymes.

The model organism E. coli was chosen for the in vivo selection for several reasons. First, E. coli is used in most lifescience laboratories and is easy to manipulate. Second, the transformation efficiency of $E$. coli with commercially available cells allows the easy generation of libraries with high complexity. While this study demonstrated the use of an E. coli library with a complexity of 9 million, it is feasible for most laboratories to increase this complexity to about 100 million. Third, while the use of a eukaryotic system may seem an advantage because the eventual application of these ribozymes would be in eukaryotic cells, the limiting factor for trans-splicing in both systems appears to be the same, and the same principles for the design of these ribozymes apply to both systems (cf. Byun et al. 2003 and Kohler et al. 1999; this study). This suggests that the initial optimization of trans-splicing ribozymes in bacterial cells and later testing of fewer ribozyme constructs in mammalian cells is a promising route to develop efficient trans-splicing ribozymes for therapeutic purposes.

The therapeutic application of transsplicing ribozymes requires them to have a high efficiency on disease-related mRNAs. However, in vivo selection procedures cannot directly optimize the ribozymes on disease-relevant mRNAs because the selection procedure requires selectable markers such as antibiotic resistance genes (Guo and Cech 2002; this study), auxotrophy (Ayre et al. 2002), or fluorescent proteins (Hasegawa et al. 2004). A solution to this problem is that regardless of the broader sequence context, specific secondary structures between the trans-splicing ribozyme and the substrate mRNA are necessary for high trans-splicing efficiency (Kohler et al. 1999; Byun et al. 2003). Therefore, structural elements that increase trans-splicing efficiency can be identified on unrelated mRNAs and later tested on disease-related mRNAs. The in vivo selection procedure can be employed in the first step of this process, screening a much larger number of ribozyme variants than could be tested individually.

The most efficient ribozyme repaired $3.8 \pm 0.5 \%$ of the substrate mRNA in bacterial cells. This is 3.8 -fold higher than the highest observed trans-splicing efficiency in bacterial cells (1\%) (Sullenger and Cech 1994). In contrast, significantly higher repair efficiencies can be achieved in mammalian cells (Jones and Sullenger 1997; Lan et al. 2000; Byun et al. 2003) for two reasons. First, the mRNA lifetime is much shorter in bacterial cells than in eukaryotic cells (Prescott 1959; Bernstein et al. 2002). This gives the ribozyme less time to fold, anneal to the substrate mRNA, and catalyze trans-splicing, and thereby reduces the total amount 


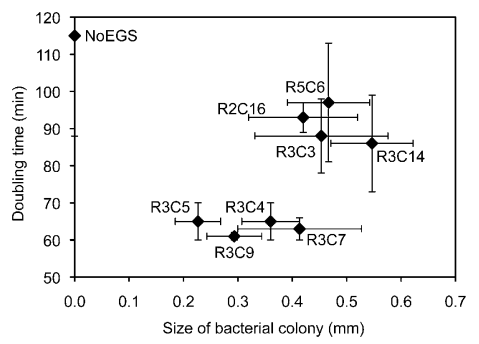

FIGURE 5. Correlation between the measurements of bacterial growth with two methods. The doubling time in liquid medium containing chloramphenicol and IPTG is shown in the $y$-axis; the diameter of bacterial colonies on LB agar plates containing IPTG and chloramphenicol is shown on the $x$-axis. The clone names are indicated. The two clusters are referred to in the text as the class with clone $\mathrm{R} 3 \mathrm{C} 14$ (upper) and the class with clone R3C7 (lower).

of trans-spliced substrate. Second, most studies in mammalian systems used stronger promoters to express the ribozymes. The resulting high ribozyme concentrations are known to increase the fraction of repaired mRNA (Jones and Sullenger 1997). Another factor that differs between an eventual application of ribozymes in therapy and our selection system is that the ribozyme and the substrate were encoded on the same plasmid molecule. This may result in partial colocalization of ribozyme and substrate, which would favor the ribozyme/substrate interactions. We hope that further studies of the selected ribozymes, as well as further selections, will successively develop group I intron ribozymes that repair a high fraction of mRNAs at low ribozyme concentrations.

\section{MATERIALS AND METHODS}

\section{Selection of splice sites}

The accessible splice sites on the chloramphenicol acetyl transferase gene were selected as described previously (Lan et al. 1998). The trans-tagging ribozyme with randomized IGS contained the sequence of the Tetrahymena group I intron ribozyme (GenBank accession no. X54512) and a 77-nt-long $3^{\prime}$-exon from the $\alpha$-mannosidase sequence from COS-7 cells (Einvik et al. 2004), using PCR primers 1 and 2 (Supplemental Table S3). The template for T7 RNA polymerase transcription of the substrate was PCR amplified from the plasmid pLysS (Novagen) in two PCRs, using primers 3 and 4 in the first PCR and then primers 5 and 6 in the second PCR. Ribozymes were transcribed from the PCR products by T7 RNA polymerase and then purified by denaturing $7 \mathrm{M}$ urea 5\% PAGE.

The reactions for the splice site selection contained $100 \mathrm{nM}$ substrate, $10 \mathrm{nM}$ ribozyme, $0.2 \mathrm{mM}$ GTP, $50 \mathrm{mM} \mathrm{MOPS} / \mathrm{KOH}$ ( $\mathrm{pH} 7.0), 135 \mathrm{mM} \mathrm{KCl}$, and $2 \mathrm{mM}$ spermidine and were incubated for $1 \mathrm{~h}$ at $37^{\circ} \mathrm{C}$. Magnesium concentrations of $5 \mathrm{mM}, 2 \mathrm{mM}$, and $1 \mathrm{mM}$ gave consistent results so that the splice sites selected under all conditions were analyzed together. Reaction products were reverse transcribed with AMV reverse transcriptase (NEB) and primer 7 (Supplemental Table S3). After RNA hydrolysis $\left(10 \mathrm{~min}\right.$ at $90^{\circ} \mathrm{C}$ in $\left.200 \mathrm{mM} \mathrm{NaOH}\right)$, reverse transcription products were PCR amplified with PCR primers 8 and 9. PCR products were cloned into the BamHI and EcoRI sites of pUC19, and sequenced to determine the splice sites.

\section{Generation of the plasmid library}

The library plasmid is based on the plasmid pUC19 with the following modification: The BamHI and SacI sites were separated by digesting pUC19 with BamHI and HindIII and then ligating with the $5^{\prime}$-phosphorylated and annealed DNA primers 10 and 11 (Supplemental Table S3), creating plasmid pUC19b. The cat gene, including its constitutive promoter and a hairpin terminator at its 3 '-end, was PCR amplified from the plasmid pLysS and ligated into pUC19b between the SacI and HindIII restriction sites. The resulting sequence of the substrate in the library plasmid is sequence 12. To create the frame shift that inactivates the cat gene, sitedirected mutagenesis with primers 13 and 14 was performed using the Stratagene quick-change protocol.

The ribozyme cassette of the library plasmid was obtained through two PCRs from a plasmid containing the sequence of the Tetrahymena group I intron. The first PCR introduced the promoter and silent mutations with primers 15 and 16. The second PCR used the primers 17 and 18. The resulting sequence encodes a ribozyme without an EGS. The promoter sequence in primers 15 and 17 was derived from plasmid pDSW204 (Weiss et al. 1999). Its -30 box is mutated from TTGACA to TTTACA to reduce ribozyme expression. The $3^{\prime}$-exon of the ribozyme was generated through PCR using the chloramphenicol acetyl transferase gene in the plasmid pLysS (Novagen) as template and primers 19 and 20. After EcoRI digest, it was joined by ligation with the EcoRI-digested ribozyme cassette. This template for the ribozyme without EGS was used to generate the six subpools with partially randomized EGSs, in two PCRs. The first PCR included the primer 21 and 22. In a second PCR, the promoters and restriction sites were attached with primers 23 and 24. The PCR products were ligated in the BamHI and SacI sites of the pUC19b plasmid containing the substrate with inactivating frame shift to generate the library plasmid. The second series of PCRs was repeated for each of the six subpools.
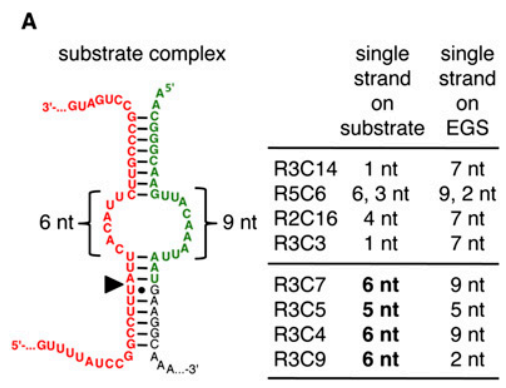

B

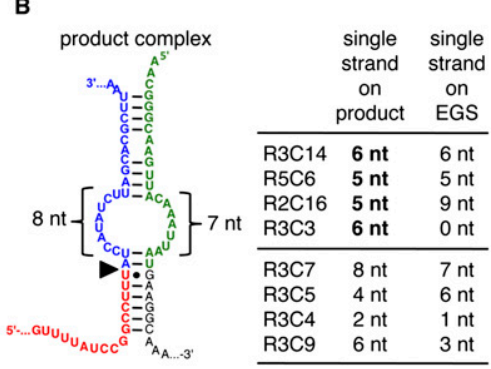

FIGURE 6. Predicted secondary structures between $(A)$ the EGS and the mRNA and $(B)$ the EGS and the trans-splicing product. As an example, the secondary structures for clone R3C7 are shown. The two tables show the number of single-stranded nucleotides in the internal loop, on the side on the mRNA substrate or the EGS. The numbers are reported for the most stable secondary structure. For the substrate complex of clone R5C6, two equally stable structures were predicted. The clones are grouped into the two classes observed for the growth behaviors (Fig. 5). Bold letters indicate that these parts of the secondary structure were conserved within each class. The mfold algorithm was used for the prediction of the structures (Zuker 2003). 


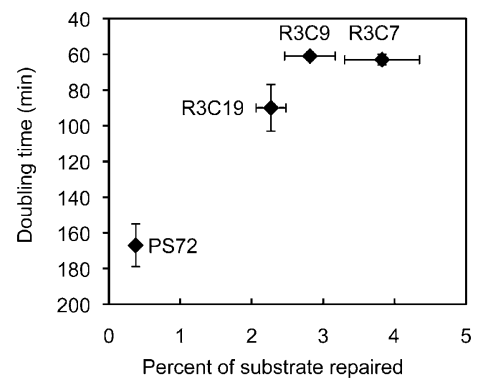

FIGURE 7. Correlation between two methods measuring in vivo trans-splicing efficiency. The percentage of repaired cat mRNA was determined by RT-PCR and is shown on the $x$-axis. The doubling time of E. coli cells expressing the respective ribozyme, in medium containing chloramphenicol, is shown on the $y$-axis. Error bars, SEM from three quantitations of the doubling time and from six qRT-PCR experiments that determined the fraction of repaired mRNA.

\section{Generation of the E. coli library}

Electrocompetent E. coli cells Stbl4 (Invitrogen) were transformed with library plasmid (1-mm slit width, $1.8 \mathrm{kV}, 25 \mu \mathrm{F}, 200 \Omega$ ), plated on SOC plates containing $100 \mathrm{mg} / \mathrm{mL}$ ampicillin medium and were incubated at $30^{\circ} \mathrm{C}$. We usually plated about 300,000 viable cells per 9-cm plate. After colonies were visible (16-20 h), the plates were washed and cells were frozen with $50 \%$ glycerol $(\mathrm{v} / \mathrm{v})$ at $-80^{\circ} \mathrm{C}$. Replating efficiencies were determined by thawing the cell stocks and plating on $\mathrm{SOC}_{\mathrm{AMP}}$ plates. In our experience, it was sufficient to calculate the replating efficiency from the $\mathrm{OD}_{600}$ of the thawed cell suspension, with $1 \mathrm{OD}_{600}$ corresponding to $2 \times 10^{8}$ cells $/ \mathrm{mL}$. The number of cells growing on the $\mathrm{SOC}_{\mathrm{AMP}}$ plates was in some cases up to twofold lower than the value expected from the $\mathrm{OD}_{600}$, presumably due to partial cell death during freeze/thawing.

To estimate the complexity of the E. coli library, we considered two factors. First, $10 \mathrm{nt}$ were randomized (Fig. 2); therefore, the maximal complexity was $4^{10}$, about 1 million. Second, the number of colonies in the $E$. coli pool with a ribozyme gene was $1.5 \times 10^{6}$ cells for each subpool. This number was obtained by multiplying the number of viable cells with the fraction of these cells that contained a ribozyme gene in the plasmid. The latter was found by colony PCR on at least 30 colonies. The fraction $\mathrm{f}$ of the initial complexity that was represented in the E. coli library, was calculated by $\mathrm{f}=1-(1-$ $(1 / \mathrm{n}))^{\mathrm{m}}$, where $\mathrm{n}$ is the initial complexity and $\mathrm{m}$ is the number of colonies with a ribozyme gene. The resulting $\mathrm{f}$ indicates that $76 \%$ of all possible pool sequences were represented in the initial pool.

We used the E. coli cell line Stbl4 (Invitrogen) in this study due to its reduced recombination frequency. However, we also performed a different selection with E. coli DH5 $\alpha$ cells. The frequency of false-positive colonies growing on chloramphenicol-containing plates was about $1 / 2000$ for E. coli $\mathrm{DH} 5 \alpha$ and about $1 / 500$ for Stbl4; therefore, we recommend the use of E. coli DH5 $\alpha$ cells for the in vivo selection system.

\section{In vivo selection}

After thawing, the E. coli subpools were mixed to give equal viable cell numbers for each subpool and shaken $1 \mathrm{~h}$ with $1 \mathrm{mM}$ IPTG at $30^{\circ} \mathrm{C}$, and then a total of 9 million clones were plated on 30 SOC plates with $8 \mu \mathrm{g} / \mathrm{mL}$ chloramphenicol and $1 \mathrm{mM}$ IPTG. After incubating for $\sim 20 \mathrm{~h}$ at $30^{\circ} \mathrm{C}$, colonies of different sizes were visible. The largest colonies $(\sim 0.8 \mathrm{~mm}$ in diameter) corresponded to false positives as judged by their ability to grow equally well on plates with different chloramphenicol concentrations. The smaller colonies (between $\sim 0.1 \mathrm{~mm}$ and $\sim 0.5 \mathrm{~mm}$ ) differed in size and number between different chloramphenicol concentrations. The cells were washed off the plates and their plasmids isolated by standard alkaline lysis and column-based purification. The ribozyme genes were purified by digesting the plasmids with SacI/ BamHI, performing electrophoresis on $1 \%$ agarose gels, excising the band with the correct size, and extracting from the gel. PCR amplification with primers 25 and 26 (Supplemental Table S3) and digestion with SacI and BamHI allowed cloning into fresh library plasmid. The high-fidelity DNA polymerase Phusion (NEB) was used for this PCR. Five rounds of selection were performed.

We estimated that each round of the selection enriched the functional ribozymes by a factor of 100-1000 because the initial complexity was $9 \times 10^{6}$ and after three rounds of selection we found duplicate sequences, among 20 sequenced clones. At five rounds of selection, few new sequences were found among the 20 sequenced clones, and one sequence was found 19 times among the 60 clones of rounds 3, 4, and 5. The enrichment factor may differ for other splice sites on cat mRNA, other antibiotic resistance genes, other cis- and trans-splicing ribozymes, and different antibiotic concentrations on the selection plates, but it illustrates the power of the in vivo selection technique.

\section{Determination of cell doubling times in liquid medium}

The doubling times of $E$. coli $\mathrm{DH} 5 \alpha$ cells that express ribozymes were determined as follows. Five-milliliter fresh overnight cultures (LB medium with $100 \mu \mathrm{g} / \mathrm{mL}$ ampicillin) were induced with $1 \mathrm{mM}$ IPTG and shaken for $1 \mathrm{~h}$ at $37^{\circ} \mathrm{C}$. The cultures were diluted to an $\mathrm{OD}_{600}$ of $0.05 \pm 0.01$ in $\mathrm{LB}$ medium with $2 \mathrm{mg} / \mathrm{mL}$ chloramphenicol and $1 \mathrm{mM}$ IPTG. Cultures were grown until the $\mathrm{OD}_{600}$ exceeded 1.0 or until the growth time reached $6 \mathrm{~h}$. The $\mathrm{OD}_{600}$ was measured after $60 \mathrm{~min}$ and every $30 \mathrm{~min}$ after. Growth rates were determined by nonlinear least squares fitting of exponential functions to the $\mathrm{OD}_{600}$ as a function of growth time. Errors were calculated from at least three independent growth curves.

\section{Measurement of bacterial colony sizes}

Bacterial colonies of E. coli DH5 $\alpha$ were grown on LB agar plates containing $8 \mu \mathrm{g} / \mathrm{mL}$ chloramphenicol and $1 \mathrm{mM}$ IPTG by incubating for $20 \mathrm{~h}$ at $37^{\circ} \mathrm{C}$. Five representative colonies and a ruler were photographed under a dissection scope equipped with a digital camera. The images of the colonies were then superimposed on the ruler to measure the colony diameters. To control for clonal variation, three cell stocks of E. coli DH5 $\alpha$ clones with the same plasmid were measured. The average of five colonies gave the value for each clone, and the average from three clones gave the reported values with corresponding standard deviations.

\section{Structure prediction}

Secondary structures were predicted on a web-based server using the mfold algorithm (Zuker 2003). We used EGSs, mRNA sequences, and $3^{\prime}$-exon sequences as shown in Figure 1 (left panel). The parameters were $37^{\circ} \mathrm{C}$ and $1 \mathrm{M} \mathrm{NaCl}$. 


\section{Quantitative RT-PCR}

Total RNA was isolated from logarithmically growing E. coli cells after induction with $1 \mathrm{mM}$ IPTG, using the RNeasy kit (Qiagen). The Qiagen RNeasy kit was used with RNAprotect Bacteria Reagent and on column DNase digestion. Three separate clones were used for each ribozyme. Two hundred nanograms of total RNA was reverse-transcribed using AMV-RT (NEB) with primer 33 for substrate, ribozyme, and product samples. The transcriptions were incubated for $1 \mathrm{~h}$ at $42^{\circ} \mathrm{C}$ with AMV reverse transcriptase (NEB). One-tenth of the reverse transcription reaction was used as template for quantitative PCR with the primers 35 and 36 for the substrate, and primers 37 and 38 for the product. The PCR was performed using the AB qPCR master mix on a Fast 7500 RT-PCR machine (Applied Biosystems). The method started with a 10-min incubation at $95^{\circ} \mathrm{C}$. Each PCR cycle included incubations of $30 \mathrm{sec}$ at $95^{\circ} \mathrm{C}, 30 \mathrm{sec}$ at $57^{\circ} \mathrm{C}$, and $30 \mathrm{sec}$ at $72^{\circ} \mathrm{C}$. The threshold cycle numbers from three eightfold serially diluted samples confirmed the linearity of the assay. No significant amount of cross-amplification was detected. Data were analyzed by least-squares fitting to an exponential function.

\section{SUPPLEMENTAL MATERIAL}

Supplemental material is available for this article.

\section{ACKNOWLEDGMENTS}

We thank Joe Pogliano, Simpson Joseph, Akif Tezcan, Lorraine Pillus, and Dario Meluzzi for helpful discussions. We thank Hector Viadiu and Thomas Hermann for critical reading of the manuscript. This work was supported by the National Institutes of Health (T32DK007233 to K.E.O.; Hemoglobin and Blood Protein Chemistry training grant to E. Komives) and by the National Science Foundation (0743985 to U.F.M.).

Received May 30, 2011; accepted December 1, 2011.

\section{REFERENCES}

Ayre BG, Kohler U, Goodman HM, Haseloff J. 1999. Design of highly specific cytotoxins by using trans-splicing ribozymes. Proc Natl Acad Sci 96: 3507-3512.

Ayre BG, Kohler U, Turgeon R, Haseloff J. 2002. Optimization of trans-splicing ribozyme efficiency and specificity by in vivo genetic selection. Nucleic Acids Res 30: e141. doi: 10.1093/nar/gnf141.

Beaudry AA, Joyce GF. 1992. Directed evolution of an RNA enzyme. Science 257: 635-641.

Bernstein JA, Khodursky AB, Lin PH, Lin-Chao S, Cohen SN. 2002. Global analysis of mRNA decay and abundance in Escherichia coli at single-gene resolution using two-color fluorescent DNA microarrays. Proc Natl Acad Sci 99: 9697-9702.

Byun J, Lan N, Long M, Sullenger BA. 2003. Efficient and specific repair of sickle $\beta$-globin RNA by trans-splicing ribozymes. RNA 9: 1254-1263.

Cadwell RC, Joyce GF. 1994. Mutagenic PCR. PCR Methods Appl 3: S136-S140.

Coetzee T, Herschlag D, Belfort M. 1994. Escherichia coli proteins, including ribosomal protein S12, facilitate in vitro splicing of phage T4 introns by acting as RNA chaperones. Genes Dev 8: $1575-1588$.

Dotson PP 2nd, Johnson AK, Testa SM. 2008. Tetrahymena thermophila and Candida albicans group I intron-derived ribozymes can catalyze the trans-excision-splicing reaction. Nucleic Acids Res 36: 5281-5289.

Einvik C, Fiskaa T, Lundblad EW, Johansen S. 2004. Optimization and application of the group I ribozyme trans-splicing reaction. Methods Mol Biol 252: 359-371.

Ellington AD, Chen X, Robertson M, Syrett A. 2009. Evolutionary origins and directed evolution of RNA. Int J Biochem Cell Biol 41: 254-265.

Fiskaa T, Birgisdottir AB. 2010. RNA reprogramming and repair based on trans-splicing group I ribozymes. New Biotechnol 27: 194-203.

Guo F, Cech TR. 2002. In vivo selection of better self-splicing introns in Escherichia coli: the role of the P1 extension helix of the Tetrahymena intron. RNA 8: 647-658.

Hasegawa S, Choi JW, Rao J. 2004. Single-cell detection of transsplicing ribozyme in vivo activity. J Am Chem Soc 126: 7158-7159.

Johnson AK, Sinha J, Testa SM. 2005. Trans insertion-splicing: ribozyme-catalyzed insertion of targeted sequences into RNAs. Biochemistry 44: 10702-10710.

Jones JT, Sullenger BA. 1997. Evaluating and enhancing ribozyme reaction efficiency in mammalian cells. Nat Biotechnol 15: $902-$ 905.

Kohler U, Ayre BG, Goodman HM, Haseloff J. 1999. Trans-splicing ribozymes for targeted gene delivery. J Mol Biol 285: 1935-1950.

Kruger K, Grabowski PJ, Zaug AJ, Sands J, Gottschling DE, Cech TR. 1982. Self-splicing RNA: autoexcision and autocyclization of the ribosomal RNA intervening sequence of Tetrahymena. Cell 31: 147-157.

Kwon BS, Jung HS, Song MS, Cho KS, Kim SC, Kimm K, Jeong JS, Kim IH, Lee SW. 2005. Specific regression of human cancer cells by ribozyme-mediated targeted replacement of tumor-specific transcript. Mol Ther 12: 824-834.

Lan N, Howrey RP, Lee SW, Smith CA, Sullenger BA. 1998. Ribozyme-mediated repair of sickle $\beta$-globin mRNAs in erythrocyte precursors. Science 280: 1593-1596.

Lan N, Rooney BL, Lee SW, Howrey RP, Smith CA, Sullenger BA. 2000. Enhancing RNA repair efficiency by combining transsplicing ribozymes that recognize different accessible sites on a target RNA. Mol Ther 2: 245-255.

Lewis MW, Wimpenny JW. 1981. The influence of nutrition and temperature on the growth of colonies of Escherichia coli K12. Can J Microbiol 27: 679-684.

Mahen EM, Harger JW, Calderon EM, Fedor MJ. 2005. Kinetics and thermodynamics make different contributions to RNA folding in vitro and in yeast. Mol Cell 19: 27-37.

Nikolcheva T, Woodson SA. 1999. Facilitation of group I splicing in vivo: misfolding of the Tetrahymena IVS and the role of ribosomal RNA exons. J Mol Biol 292: 557-567.

Ohuchi SJ, Ikawa Y, Shiraishi H, Inoue T. 2004. Artificial modules for enhancing rate constants of a Group I intron ribozyme without a P4-P6 core element. J Biol Chem 279: 540-546.

Prescott DM. 1959. Nuclear synthesis of cytoplasmic ribonucleic acid in Amoeba proteus. J Biophys Biochem Cytol 6: 203-206.

Reyrolle J, Letelier F. 1979. Autoradiographic study of the localization and evolution of growth zones in bacterial colonies. J Gen Microbiol 111: 399-406.

Ryu KJ, Kim JH, Lee SW. 2003. Ribozyme-mediated selective induction of new gene activity in hepatitis $\mathrm{C}$ virus internal ribosome entry site-expressing cells by targeted trans-splicing. Mol Ther 7: 386-395.

Sullenger BA, Cech TR. 1994. Ribozyme-mediated repair of defective mRNA by targeted, trans-splicing. Nature 371: 619-622.

Treiber DK, Rook MS, Zarrinkar PP, Williamson JR. 1998. Kinetic intermediates trapped by native interactions in RNA folding. Science 279: 1943-1946.

Weiss DS, Chen JC, Ghigo JM, Boyd D, Beckwith J. 1999. Localization of FtsI (PBP3) to the septal ring requires its membrane anchor, the $\mathrm{Z}$ ring, FtsA, FtsQ, and FtsL. J Bacteriol 181: 508-520.

Zuker M. 2003. Mfold web server for nucleic acid folding and hybridization prediction. Nucleic Acids Res 31: 3406-3415. 

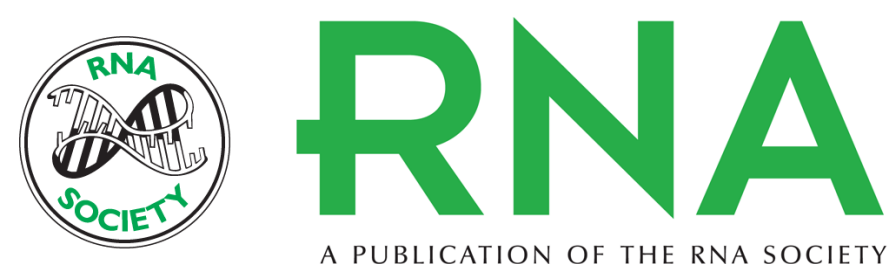

A PUBLICATION OF THE RNA SOCIETY

\section{An in vivo selection method to optimize trans-splicing ribozymes}

Karen E. Olson and Ulrich F. Müller

RNA 2012 18: 581-589 originally published online January 24, 2012

Access the most recent version at doi:10.1261/rna.028472.111

Supplemental

Material

References

\section{License}

Email Alerting Service
http://rnajournal.cshlp.org/content/suppl/2012/01/03/rna.028472.111.DC1

This article cites 31 articles, 11 of which can be accessed free at: http://rnajournal.cshlp.org/content/18/3/581.full.html\#ref-list-1 